\title{
Retrograde Versus Antegrade Approach for the Endovascular Treatment of Symptomatic Femoropopliteal Disease: Results of the 2-Year Follow Up
}

\author{
Emced Khalil, ${ }^{1}$ Sedat Ozcan ${ }^{2}$ \\ ${ }^{1}$ Department of Cardiovascular Surgery, Ordu University Research and Education Hospital, Ordu, Turkey; ${ }^{2}$ Department of \\ Cardiovascular Surgery, Çanakkale 18 Mart University Faculty of Medicine, Çanakkale, Turkey
}

\section{ABSTRACT}

Objective: To compare the antegrade and retrograde approaches, in terms of access site complications and longterm patency in subjects undergoing EVT for symptomatic SFA or popliteal artery stenosis or occlusions.

Methods: All consecutive patients who underwent retrograde recanalization for partial stenosis or chronic total occlusion of the SFA or PA at 2 centers were enrolled in this retrospective analysis. Subjects were divided into 2 groups, according to the approach selected for EVT as antegrade approach group or retrograde approach group. The rate of primary patency, which was defined as the lack of restenosis at the target lesion, was the primary outcome measure, during the follow-up evaluations. The rate of procedural complications, including hematoma, bleeding, and distal embolism, were secondary outcome measures.

Results: A significant improvement occurred in ABI, following the intervention in both the antegrade approach group [0.7 (0.3 - 1.1) versus $0.85(0.4-1.3), \mathrm{P}<0.001]$ and retrograde approach group $[0.5(0.3-1.1)$ versus $0.8(0.3-1.3), P<$ $.001]$. The primary patency rate at the $1 \mathrm{st}, 6 \mathrm{th}, 12$ th and 24 th months of the intervention in the antegrade approach group were $94.85 \%, 83.82 \%, 74.26 \%$, and $66.91 \%$, respectively. The primary patency rate at the 1st, 6th, 12th, and 24th months of the intervention in the retrograde approach group were $93.33 \%, 86.67 \%, 84.44 \%$, and $71.11 \%$, respectively. The groups were similar with respect to the primary patency rates. The rate of complications, including hematoma, bleeding, and distal embolization was similar in the 2 groups.

Conclusion: Antegrade approach and retrograde approach provide a similar safety profile in the EVT of SFA and popliteal artery stenosis and occlusion. The primary patency rates at the 1st, 6th, 12th, and 24th months of follow up also were similar in the 2 groups. However, the significant difference in the lesion characteristics of the subjects undergoing retrograde or antegrade approach complicates the ability to reach a clear conclusion, regarding the superiority of one technique over the other.

Received April 9, 2020; accepted April 27, 2020.

Correspondence: Emced Khalil, Department of Cardiovascular Surgery, Ordu University Research and Education Hospital, Ordu, Turkey. (e-mail: emjedkbalil@gmail.com).

\section{INTRODUCTION}

Atherosclerotic peripheral artery disease affects about $25 \%$ of the adult population $>65$ years old. Superficial femoral artery (SFA) stenosis or occlusions are responsible for more than $50 \%$ of the cases with claudication [Wojtasik-Bakalarz 2017]. Endovascular treatment (EVT) constitutes a safe and viable option for the revascularization of the subjects with TASC A-C lesions; nevertheless, surgery should be taken into consideration in TASC-D lesions [Perera 2007].

Endovascular treatment increasingly is employed in the treatment of symptomatic high-grade stenosis or occlusions of the superficial femoral artery and popliteal artery owing to the less-invasive nature of the EVT and similar outcomes compared with the surgical revascularization [Marone 2018; Powell 2008]. The high restenosis rate following the intervention was the main limiting factor for EVT; however, implementation of the drug coated balloons led to a significant decline in restenosis rates and improved primary patency rate [Schneider 2018; Rosenfield 2015; Miller 2017; Niazi 2017].

Contralateral retrograde puncture or ipsilateral antegrade puncture of the common femoral artery frequently are employed when performing antegrade approach EVT [Schneider 2017]. However, antegrade puncture is not feasible in case of a blunted, calcified proximal cap, which makes guidewire passage complicated, and in subjects with a proximal SFA disease, in whom sheath replacement is problematic due to the short distance between the access point and the proximal cap of the SFA lesion [Schmieder 2008; Stavroulakis 2019]. Popliteal artery puncture, which makes the use of lower profile sheaths and devices possible, recently has gained popularity as an alternative to the antegrade approach, particularly due to reportedly reduced risks of vascular complications at the access site [Ueshima 2015].

Despite several reports demonstrating the safety and reliability of the retrograde popliteal approach in EVT of the SFA and popliteal artery, data comparing the long-term results of the antegrade and retrograde approaches in these patients is limited. This study aimed to compare the antegrade and retrograde approaches, in terms of access site complications and long-term patency in subjects undergoing EVT for symptomatic SFA or popliteal artery stenosis or occlusions. 


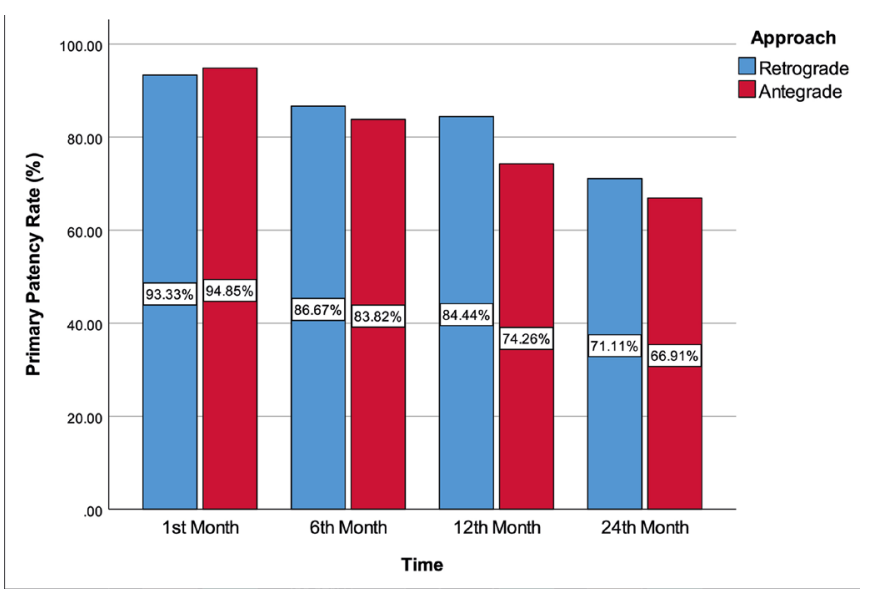

Figure 1. Ankle-brachial index with regard to approach used for the arterial access.

\section{MATERIALS AND METHODS}

All consecutive patients who underwent retrograde recanalization for partial stenosis or chronic total occlusion of the SFA or PA at Ordu Training and Research Hospital and Gaziantep University Hospital between August 2017 and May 2019 were enrolled in this retrospective study. SFA or PFA disease was confirmed by CT angiography in subjects with risk factors for atherosclerotic vascular disease and symptoms of stenotic peripheral arterial disease, including rest pain, claudication, and cold lower extremities. Digital subtraction angiography was performed for a detailed description of lesion characteristics and identification of the collateral blood supply prior to the intervention. Subjects with a history of prior SFA or PA intervention or surgery, additional iliac artery and abdominal aorta stenosis, previous coronary artery bypass grafting surgery, and those with contraindications to antiaggregant agents were excluded from the study. All required data were retrieved from the institutional digital database and patient charts. Written informed consent was obtained from all participants. The study was approved by the Institutional Review Board and was conducted in accordance with the Helsinki declaration. Subjects were divided into 2 groups, according to the approach selected for EVT, as the antegrade approach group or retrograde approach group. The main indications for a retrograde approach were severe calcification and/or relatively long CTO segment on CT angiography and failed antegrade approach attempt.

Endovascular treatment: The interventional procedures were carried out by the same interventional team of the relevant center dedicated to peripheral artery diseases. Before the intervention, $100 \mathrm{U} / \mathrm{kg}$ UF heparin was administered, and arterial puncture was done under local anesthesia. Ipsilateral common femoral artery puncture was carried out with a 20 or $22 \mathrm{G}$ needle under ultrasonographic guidance with the patient in the supine position. In patients undergoing EVT with the retrograde approach, popliteal artery puncture was carried out with a 20 or $22 \mathrm{G}$ needle with the patient in the prone position. Five to seven French sheaths were placed

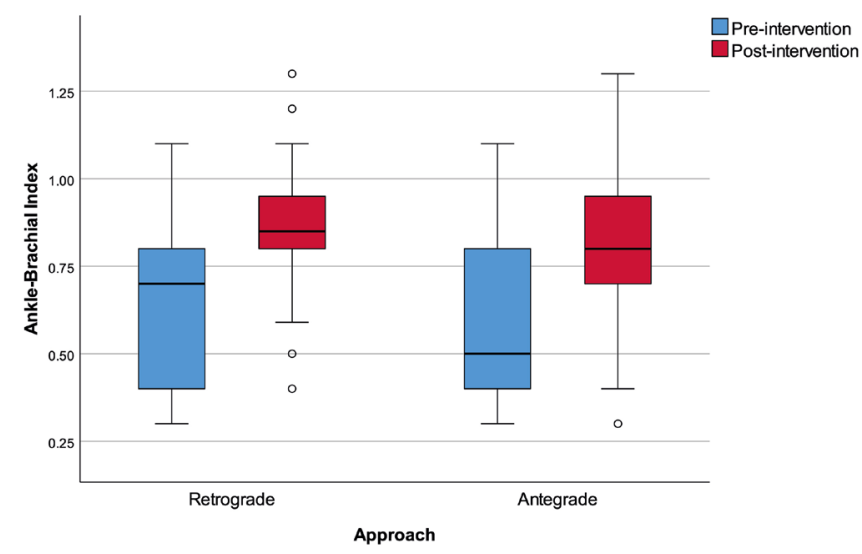

Figure 2. Primary patency rates of the subjects undergoing endovascular treatment with antegrade or retrograde approach.

into the artery. Stenotic lesions and occlusions were crossed using 0.035 inches hydrophilic guidewires. Over-the-wire paclitaxel-coated balloons, which were compatible with the guidewire, were inflated at the target lesion for at least 2 minutes. The decision for stent implantation was left to the operator's discretion. Vascular sheaths were left in the artery for 4 hours after the procedure and then were removed. Hemostasis was achieved by manual compression, during sheath removal. Patients were treated by dual antiplatelet therapy (acetylsalicylic acid and clopidogrel) for 6 months after the procedure.

Primary outcome: All subjects underwent physical examination and color duplex ultrasound at the 1st, 6th, 12 th, and 24th months of the intervention to evaluate the patency of the target vessel. The rate of primary patency, which was defined as the lack of restenosis at the target lesion, was the primary outcome measure, during the follow-up evaluations. The rate of procedural complications, including hematoma, bleeding, and distal embolism, were secondary outcome measures.

Statistical analysis: All analyses were performed on SPSS v21 (SPSS Inc., Chicago, IL). Shapiro-Wilk test was used for determining whether variables are normally distributed. Data are given as mean \pm standard deviation or median (minimum - maximum) for continuous variables with regard to normality of distribution and frequency (percentage) for categorical variables. Normally distributed variable (age) were analyzed with the independent samples t test. Non-normally distributed variables (length of stenosis) were analyzed with the Mann Whitney $U$ test. The change in Ankle-brachial index during follow up was analyzed with the Wilcoxon Signed Ranks test for repeated measurements. Between groups, comparison of the ankle-brachial index was performed by analyzing differences between measurements with the Mann Whitney $U$ test. Categorical variables evaluated by using Chi-square test or Fisher's exact test. Generalized estimating equations were used for analyzing primary patency rates between groups. Repeated measurements of primary patency rate were analyzed with the Cochran's Q test. Pairwise comparisons were performed with the Bonferroni correction method. Two tailed $P$-values of less than 0.05 were considered statistically significant. 
Table 1. Summary of Patient Characteristics

\begin{tabular}{|c|c|c|c|}
\hline & \multicolumn{2}{|c|}{ Approach } & \multirow[b]{2}{*}{$P$} \\
\hline & $\begin{array}{l}\text { Retrograde } \\
(N=45)\end{array}$ & $\begin{array}{l}\text { Antegrade } \\
(N=136)\end{array}$ & \\
\hline Age (years) & $63.42 \pm 11.54$ & $61.67 \pm 11.89$ & .391 \\
\hline Gender (male) & $40(88.89 \%)$ & $118(86.76 \%)$ & .910 \\
\hline \multicolumn{4}{|l|}{ Rutherford Classification } \\
\hline 3 & $21(46.67 \%)$ & $66(48.53 \%)$ & \\
\hline 4 & $22(48.89 \%)$ & $39(28.68 \%)$ & .006 \\
\hline 5 & $2(4.44 \%)$ & $31(22.79 \%)$ & \\
\hline \multicolumn{4}{|l|}{ TASC ॥ } \\
\hline A & $0(0.00 \%)$ & $2(1.47 \%)$ & \\
\hline B & $28(62.22 \%)$ & $69(50.74 \%)$ & .395 \\
\hline C & $11(24.44 \%)$ & $34(25.00 \%)$ & \\
\hline D & $6(13.33 \%)$ & $31(22.79 \%)$ & \\
\hline Smokers & $16(35.56 \%)$ & $58(42.65 \%)$ & .507 \\
\hline Hyperlipidaemia & $15(33.33 \%)$ & $53(38.97 \%)$ & .618 \\
\hline Diabetes Mellitus & $18(40.00 \%)$ & $70(51.47 \%)$ & .245 \\
\hline Hypertension & $34(75.56 \%)$ & 79 (58.09\%) & .055 \\
\hline Chronic Renal Failure & $2(4.44 \%)$ & $10(7.35 \%)$ & .733 \\
\hline COPD & $7(15.56 \%)$ & $6(4.41 \%)$ & .019 \\
\hline Coronary Artery Disease & $14(31.11 \%)$ & $31(22.79 \%)$ & .358 \\
\hline \multicolumn{4}{|l|}{ Indication } \\
\hline Claudication & $18(40.00 \%)$ & $71(52.21 \%)$ & \\
\hline Rest Pain & $27(60.00 \%)$ & $64(47.06 \%)$ & .289 \\
\hline Wound & & $0(0.00 \%)$ & $\begin{array}{c}1 \\
(.74 \%)\end{array}$ \\
\hline
\end{tabular}

Data are given as mean \pm standard deviation and as frequency (percentage) for categorical variables. COPD: Chronic Obstructive Pulmonary Disease

\section{RESULTS}

Longitudinal data was available for 181 subjects (mean age $62.11 \pm 11.80$ years, $87.2 \%$ male). The antegrade approach was used in 136 subjects, and the retrograde approach was used in 45 subjects. Patient characteristics are presented in Table 1. The Rutherford classes of the subjects receiving the antegrade approach were higher than that of the subjects undergoing EVT with the retrograde approach. However, the 2 groups were similar with respect to the TASC classification. The groups also were similar regarding the rate of the comorbid diseases except for the presence of chronic obstructive pulmonary disease, which significantly was more frequent in the retrograde approach group compared with the antegrade approach group.

Table 2 demonstrates lesion characteristics. Subjects undergoing EVT with the retrograde approach more
Table 2. Angiographic characteristics

\begin{tabular}{|c|c|c|c|}
\hline & \multicolumn{2}{|c|}{ Approach } & \multirow[b]{2}{*}{$P$} \\
\hline & $\begin{array}{l}\text { Retrograde } \\
(\mathrm{N}=45)\end{array}$ & $\begin{array}{l}\text { Antegrade } \\
(\mathrm{N}=136)\end{array}$ & \\
\hline Length of Stenosis (cm) & $10(4-40)$ & $10(3-25)$ & .096 \\
\hline Directional Atherectomy & $18(40.00 \%)$ & $18(13.33 \%)$ & $<.001$ \\
\hline \multicolumn{4}{|c|}{ Lesion Characteristic (Occlusion) } \\
\hline Partial (>70\% stenosis) & $14(31.11 \%)$ & $97(71.32 \%)$ & $<.001$ \\
\hline Total occlusion & $31(68.89 \%)$ & $39(28.68 \%)$ & \\
\hline \multicolumn{4}{|l|}{ Localization } \\
\hline SFA & $1(2.22 \%)$ & $3(2.21 \%)$ & \\
\hline Proximal SFA & $22(48.89 \%)$ & $7(5.15 \%)$ & \\
\hline Mid SFA & $4(8.89 \%)$ & 38 (27.94\%) & $<.001$ \\
\hline Distal SFA & $1(2.22 \%)$ & $49(36.03 \%)$ & \\
\hline SFA + POPL & $17(37.78 \%)$ & $39(28.68 \%)$ & \\
\hline \multicolumn{4}{|l|}{ Length of Balloon (mm) } \\
\hline 40 & $1(2.33 \%)$ & $4(2.96 \%)$ & \\
\hline 60 & $5(11.63 \%)$ & $19(14.07 \%)$ & \\
\hline 80 & $8(18.60 \%)$ & $32(23.70 \%)$ & .681 \\
\hline 100 & $14(32.56 \%)$ & $29(21.48 \%)$ & \\
\hline 150 & $15(34.88 \%)$ & $51(37.78 \%)$ & \\
\hline \multicolumn{4}{|l|}{ Diameter of Balloon (mm) } \\
\hline$\leq 4$ & $12(27.91 \%)$ & $57(42.22 \%)$ & \\
\hline 5 & $21(48.84 \%)$ & $63(46.67 \%)$ & .075 \\
\hline$\geq 6$ & $10(23.26 \%)$ & $15(11.11 \%)$ & \\
\hline \multicolumn{4}{|l|}{ Sheath } \\
\hline $6 \mathrm{~F}$ & $25(56.82 \%)$ & $20(14.71 \%)$ & \\
\hline $7 F$ & $19(43.18 \%)$ & $116(85.29 \%)$ & $<.001$ \\
\hline Microcatheter alone & $14(31.11 \%)$ & $4(2.94 \%)$ & $<.001$ \\
\hline
\end{tabular}

Data are given as median (minimum - maximum) for continuous variables with regard to normality of distribution and as frequency (percentage) for categorical variables

frequently received directional atherectomy compared with those undergoing EVT with the antegrade approach. In addition, lesions with total occlusion were more frequent in the retrograde approach group compared with the antegrade approach group. While proximal SFA lesions were more frequent in the antegrade approach group, the rate of mid and distal SFA lesions were higher in the retrograde approach group.

Primary and secondary outcomes of the 2 groups are represented in Table 3. The rate of complications, including hematoma, bleeding and distal embolization were similar in the 2 groups. Pre- and post-interventional ankle brachial index (ABI) of the 2 groups were similar. A significant improvement occurred in $\mathrm{ABI}$, following the intervention 
Table 3. Primary and secondary outcomes

\begin{tabular}{|c|c|c|c|}
\hline & \multicolumn{2}{|c|}{ Approach } & \multirow[b]{2}{*}{$P$} \\
\hline & $\begin{array}{l}\text { Retrograde } \\
(\mathrm{N}=45)\end{array}$ & $\begin{array}{l}\text { Antegrade } \\
(N=136)\end{array}$ & \\
\hline Complications & $3(6.67 \%)$ & $3(2.21 \%)$ & .164 \\
\hline Hematoma & $1(2.22 \%)$ & $2(1.47 \%)$ & \\
\hline Bleeding & $1(2.22 \%)$ & $0(0.00 \%)$ & \\
\hline Embolism & $1(2.22 \%)$ & $1(0.74 \%)$ & \\
\hline \multicolumn{4}{|c|}{ Ankle-Brachial Index } \\
\hline Pre-intervention & $0.7(0.3-1.1)$ & $0.5(0.3-1.1)$ & \\
\hline Post-intervention & $0.85(0.4-1.3)$ & $0.8(0.3-1.3)$ & .506 \\
\hline $\mathrm{P}$ (within groups) & $<0.001$ & $<0.001$ & \\
\hline \multicolumn{4}{|c|}{ Primary patency rate } \\
\hline 1st month & $42(93.33 \%)$ a & $129(94.85 \%) \mathrm{a}$ & \\
\hline 6th month & $39(86.67 \%) a b$ & $114(83.82 \%) a b$ & \\
\hline 12th month & $38(84.44 \%) a b$ & $101(74.26 \%) b c$ & .423 \\
\hline 24th month & $32(71.11 \%) b$ & $91(66.91 \%) c$ & \\
\hline P (within groups) & 0.002 & $<0.001$ & \\
\hline \multicolumn{4}{|c|}{ Additional information } \\
\hline Re-intervention & $8(61.54 \%)$ & $45(80.36 \%)$ & .162 \\
\hline Surgery & $4(30.77 \%)$ & $4(7.14 \%)$ & .036 \\
\hline Amputation & $0(0.00 \%)$ & $4(7.14 \%)$ & 1.000 \\
\hline Exitus & $1(7.69 \%)$ & $1(1.79 \%)$ & .344 \\
\hline Lost to Follow up & $0(0.00 \%)$ & $4(7.14 \%)$ & 1.000 \\
\hline
\end{tabular}

Data are given as median (minimum - maximum) for continuous variables with regard to normality of distribution and as frequency (percentage) for categorical variables.

Same letters denote the lack of statistically significant difference between repeated measurements.

in both the antegrade approach group [0.7 $(0.3-1.1)$ versus $0.85(0.4-1.3), P<.001]$ and retrograde approach group [0.5 (0.3 - 1.1) versus $0.8(0.3-1.3), P<.001]$ (Figure 1$)$. The primary patency rate at the 1st, 6th, 12th and 24th months of the intervention in the antegrade approach group were $94.85 \%, 83.82 \%, 74.26 \%$, and $66.91 \%$, respectively. The primary patency rate at the 1st, 6th, 12th and 24th months of the intervention in the retrograde approach group were $93.33 \%, 86.67 \%, 84.44 \%$, and $71.11 \%$, respectively. The groups were similar with respect to the primary patency rates. (Figure 2) Re-intervention was required in $62.54 \%$ of the subjects in the retrograde approach group and in $80.36 \%$ of the subjects in the antegrade approach group $(P=0.162)$. Cardiovascular mortality and the rate of amputation were similar in the 2 groups. Subjects receiving VT with the retrograde approach more frequently underwent surgery compared with those receiving EVT with the antegrade approach.

\section{DISCUSSION}

This study demonstrated that the retrograde approach in the EVT of subjects with SFA and popliteal artery stenosis or occlusions provides favorable results, in terms of safety and long-term reliability which is comparable to that achieved with the antegrade approach. Both techniques provide significant increase in the post-interventional $\mathrm{ABI}$ and similar mid- and long-term patency rates. Moreover, access site complications, including local hematoma, bleeding, and distal embolism are similar in the 2 groups.

Lower extremity peripheral arterial disease currently is the third cause of death from cardiovascular diseases, following coronary artery disease and stroke [Murakami 2018]. Subsequent to the introduction of the endovascular solution for the treatment of the lower extremity peripheral arterial disease, the strategy of revascularization has shifted from surgical treatment toward EVT [Goodney 2009]. The SFA commonly is affected by symptomatic peripheral arterial disuse. EVT is recommended even in symptomatic TASC-C and D type lesions of the SFA [Marone 2018]. The main drawback and Achilles heel of percutaneous techniques of SFA and popliteal artery EVT, restonosis, has been overcome by the use of drug coated technologies such as drug eluting stents and drug coated balloons [Drachman 2019].

Endovascular treatment of SFA and popliteal artery lesions usually is managed by the antegrade approach through contralateral retrograde puncture or ipsilateral antegrade puncture of the common femoral artery [Powell 2008]. However, crossing the lesion in the presence of a total occlusion may be challenging in $10 \%$ to $20 \%$ of the cases, due to the blunted stump and severe calcification [Pua 2015]. Therefore, retrograde access through popliteal artery puncture may stand as a reliable alternative to the antegrade access in subjects with failed antegrade approach or in the presence of poor lesion characteristics [Fanelli 2011; Noory 2009; Tokuda 2014]. Recent studies investigating the utility of retrograde approach in the management of SFA or popliteal artery have revealed that popliteal artery approach provides a higher primary success rate compared with the non-popliteal approach, but similar total complication rate and major complication rate [Ueshima 2015]. One-year patency rate of the SFA chronic total occlusions managed with the use of the retrograde popliteal artery access has been reported as 85.7 in a recent study conducted by Dumantepe et al. [Dumantepe 2017]. However, the number of studies comparing the antegrade and retrograde approaches is limited, and the study population enrolled in the previous studies is relatively small.

From this point of view, our findings may improve the current knowledge regarding the utility of retrograde approach in symptomatic SFA and popliteal artery atherosclerotic disease. Two centers and a total of 181 subjects were included in this retrospective analysis. We could reach 24 months followup data in this population, and the number of subjects losing follow-up was extremely low. The sheath size was lower in subjects receiving the retrograde approach; however, the rate of local complications was similar to that of the antegrade approach. In other words, despite the insertion of larger 
sheaths in the antegrade approach group, the rate of local complications were similar to that of the retrograde approach. The improvement in primary patency rate and ABI was similar in the 2 approaches. These findings show that antegrade approach is at least non-inferior to the retrograde approach, in terms of the safety profile. However, differences in lesion characteristics, including the location of the lesion and the higher rate of chronic total occlusions in subjects receiving retrograde approach makes it impossible to reach a clear conclusion, regarding the superiority of one technique over the other with respect to the primary patency rate.

With this in mind, we speculate that antegrade approach in the EVT of the SFA and popliteal artery peripheral artery disease provides at least similar safety to that of the retrograde approach, in terms of access site complications. Improvement in post-interventional $\mathrm{ABI}$ and the primary patency rates at the 1st, 6 th, 12 th, and 24th months of the follow up was similar in the 2 groups. However, given the higher rate of chronic total occlusions in subjects receiving retrograde approach compared with those receiving antegrade approaches, this study is not sufficient to conclude regarding the primary patency rates. This is a major limitation for this study. These results therefore need to be interpreted with caution. Further randomized, prospective studies are required to compare the antegrade and retrograde approach in the EVT of the subjects with SFA or popliteal artery disease, regarding the primary patency rate.

\section{CONCLUSION}

In conclusion, antegrade approach and retrograde approach provide a similar safety profile in the EVT of SFA and popliteal artery stenosis and occlusion. The primary patency rates at the 1 st, 6th, 12 th, and 24th months of the follow up also were similar in the 2 groups. However, the significant difference in the lesion characteristics of the subjects undergoing retrograde or antegrade approach complicates reaching a clear conclusion, regarding the superiority of one technique over the other. Further randomized, prospective studies are required to answer this question.

\section{REFERENCES}

Drachman DE. 2019. Endovascular Treatment of the Superficial Femoral Artery: When Nothing Is Actually Quite Something. J Am Coll Cardiol 73(6):680-3.

Dumantepe M. 2017. Retrograde Popliteal Access to Percutaneous Peripheral Intervention for Chronic Total Occlusion of Superficial Femoral Arteries. Vascular and endovascular surgery. 51(5):240-6.

Fanelli F, Lucatelli P, Allegritti M, Corona M, Rossi P, Passariello R. 2011. Retrograde popliteal access in the supine patient for recanalization of the superficial femoral artery: initial results. Journal of endovascular therapy: an official journal of the International Society of Endovascular Specialists. 18(4):503-9.

Goodney PP, Beck AW, Nagle J, Welch HG, Zwolak RM. 2009. National trends in lower extremity bypass surgery, endovascular interventions, and major amputations. Journal of vascular surgery. 50(1):54-60.

Marone EM, Rinaldi LF, Argenteri A, Chiesa R. 2018. Current treatment of superficial femoral artery disease: a national survey. J Cardiovasc Surg (Torino). 59(5):755-6.

Miller AJ, Takahashi EA, Harmsen WS, Mara KC, Misra S. 2017. Treatment of Superficial Femoral Artery Restenosis. Journal of vascular and interventional radiology: JVIR. 28(12):1681-6.

Murakami A. 2018. Towards the Application of Endovascular Treatment for Superficial Femoral Artery. Circ J. 82(5):1253-4.

Niazi K, Tukaye DN. 2017. Challenges of Stent Restenosis in Superficial Femoral and Popliteal Artery Disease. JACC Cardiovasc Interv. 10(20):2124-5.

Noory E, Rastan A, Schwarzwalder U, Sixt S, Beschorner U, Burgelin K, et al. 2009. Retrograde transpopliteal recanalization of chronic superficial femoral artery occlusion after failed re-entry during antegrade subintimal angioplasty. Journal of endovascular therapy: an official journal of the International Society of Endovascular Specialists. 16(5):619-23.

Perera GB, Lyden SP. 2007. Current trends in lower extremity revascularization. Surgical Clinics of North America. 87(5):1135-47.

Powell RJ. 2008. Endovascular treatment in the superficial femoral artery: which devices, where? Seminars in vascular surgery. 21(4):180-5.

Pua U. 2015. Profunda anchor technique for ipsilateral antegrade approach in endovascular treatment of superficial femoral artery ostial occlusion. Cardiovasc Intervent Radiol. 38(2):453-6.

Rosenfield K, Jaff MR, White CJ, Rocha-Singh K, Mena-Hurtado C, Metzger DC, et al. 2015. Trial of a paclitaxel-coated balloon for femoropopliteal artery disease. New England Journal of Medicine. 373(2):145-53.

Schmieder GC, Panneton JM. 2008. Endovascular superficial femoral artery treatment: can it be as good as bypass? Seminars in vascular surgery. 21(4):186-94.

Schneider PA. 2017. Evolution and current use of technology for superficial femoral and popliteal artery interventions for claudication. J Vasc Surg. 66(3):916-23.

Schneider PA, Laird JR, Tepe G, Brodmann M, Zeller T, Scheinert D, et al. 2018. Treatment effect of drug-coated balloons is durable to 3 years in the femoropopliteal arteries: long-term results of the IN. PACT SFA randomized trial. Circulation: Cardiovascular Interventions. 11(1):e005891.

Stavroulakis K, Argyriou A, Watts M, Varghese JJ, Estes BA, Torsello G, et al. 2019. How to deal with calcium in the superficial femoral artery. J Cardiovasc Surg (Torino). 60(5):572-81.

Tokuda T, Hirano K, Muramatsu T, Tsukahara R, Nakano M. 2014. A sheathless retrograde approach via the popliteal artery is useful and safe for treating chronic total occlusions in the superficial femoral artery. Journal of endovascular therapy: an official journal of the International Society of Endovascular Specialists. 21(2):289-95.

Ueshima D, Ashikaga T, Shimura T, Hatano Y, Sasaoka T, Kurihara K, et al. 2015. Popliteal retrograde approach is effective and safe for superficial femoral artery chronic total occlusion. Annals of Vascular Diseases. 8(3):220-6.

Wojtasik-Bakalarz J, Arif S, Chyrchel M, Rakowski T, Bartus K, Dudek D, et al. 2017. Twelve months follow-up after retrograde recanalization of superficial femoral artery chronic total occlusion. Postepy Kardiol Interwencyjnej. 13(1):47-52. 